\title{
Trigonometric quark confinement potential of QCD traits
}

\author{
C.B. Compean and M. Kirchbach ${ }^{\mathrm{a}}$ \\ Instituto de Fisica, UASLP, Av. Manuel Nava 6, Zona Universitaria, San Luis Potosi, SLP 78290, Mexico
}

Received: 30 May 2007 / Revised: 11 July 2007

Published online: 31 July 2007 - C Società Italiana di Fisica / Springer-Verlag 2007

Communicated by A. Schäfer

\begin{abstract}
We make the case that the Coulomb- plus linear quark confinement potential predicted by lattice QCD is an approximation to the exactly solvable trigonometric Rosen-Morse potential that has the property to interpolate between the Coulomb- and the infinite wells. We test the predictive power of this potential in the description of the nucleon (considered as a quark-diquark system) and provide analytic expressions for its mass spectrum and the proton electric form factor. We compare the results obtained in this fashion to data and find quite good agreement. We obtain an effective gluon propagator in closed form as the Fourier transform of the potential under investigation.
\end{abstract}

PACS. 12.39.Jh Non-relativistic quark models - 13.40.Gp Electromagnetic form factors

\section{The quark potential from lattice QCD}

The strong interactions of quarks, the fundamental constituents of hadrons, are governed by the Quantum Chromodynamics (QCD), the non-Abelian gauge theory with the gluons as gauge bosons. As a consequence of the non-Abelian character of QCD, the quark interactions run from one- to many-gluon exchanges over gluon selfinteractions, the latter being responsible for the so-called quark confinement, where highly energetic quarks remain trapped but behave as (asymptotically) free particles at high energies and momenta. The QCD equations are nonlinear and complicated due to the gluon self-interaction processes and their solution requires employment of highly sophisticated techniques such as discretization of space time, so-called lattice QCD. Lattice QCD calculations have established themselves as a reliable tool for the non-perturbative analysis of QCD. The outcome (in the quenched approximation) is a linear confinement potential with energy increase, be it quark-anti-quark $(Q \bar{Q})$,

$$
V_{Q \bar{Q}}(|\mathbf{r}|)=-\frac{A_{Q \bar{Q}}}{|\mathbf{r}|}+\sigma_{Q \bar{Q}}|\mathbf{r}|+C_{Q \bar{Q}}
$$

or two-body potential between quarks (so-called $\Delta$ type),

$V_{3 Q}\left(\mathbf{r}_{1}, \mathbf{r}_{2}, \mathbf{r}_{3}\right)=-A_{\Delta} \sum_{i<j} \frac{1}{\left|\mathbf{r}_{i}-\mathbf{r}_{j}\right|}+\sigma_{\Delta} \sum_{i<j}\left|\mathbf{r}_{i}-\mathbf{r}_{j}\right|+C_{\Delta}$,

\footnotetext{
a e-mail: mariana@ifisica.uaslp.mx
}

in obvious notations. The Coulomb-like piece is associated with short-range one-gluon exchange in the perturbative regime, whereas the long-range linear part relates to nonperturbative effects and is attributed to flux-tube $Q \bar{Q}$, or $Q Q$ links. Its strength is then associated with the respective string tension [1]. Detailed analysis of the values of the constants of $V_{Q \bar{Q}}$ and $V_{3 Q}$ has been performed in ref. [2]. The three-quark (3Q) Coulomb- plus linear potential, or, versions of it, has found repeatedly application to baryon spectroscopy $[3,4]$. In view of this, its generalization to an exactly solvable potential is of interest.

We begin with first drawing attention to the proximity of the two-body Coulomb- plus linear potential (be it for $Q \bar{Q}, Q Q$, or $Q(Q Q)$ systems), to $(-\cot |\mathbf{r}|)$. Indeed, this is immediately seen from the corresponding Taylor expansion,

$$
-\cot |\mathbf{r}| \approx-\frac{1}{|\mathbf{r}|}+\frac{1}{3}|\mathbf{r}|
$$

This expression shows that the absolute values of the strengths of the linear to Coulomb potentials are in ratio $1: 3$, a value that fits quite reasonably into the range of the $\sigma_{\Delta}: A_{\Delta}$ ratio of approximately $1 / 4-1 / 2$ reported by the lattice QCD analysis [2]. In fact, $(-\cot |\mathbf{r}|)$ is part of the more general and exactly solvable trigonometric potential

$$
v_{\mathrm{tRM}}(|\mathbf{z}|)=-2 b \cot |\mathbf{z}|+a(a+1) \csc ^{2}|\mathbf{z}|, \quad \mathbf{z}=\frac{\mathbf{r}}{d},
$$

known in supersymmetric quantum mechanics (SUSYQM) under the name of the trigonometric Rosen-Morse potential [5] and displayed in fig. 1. The potential in eq. (4) is 


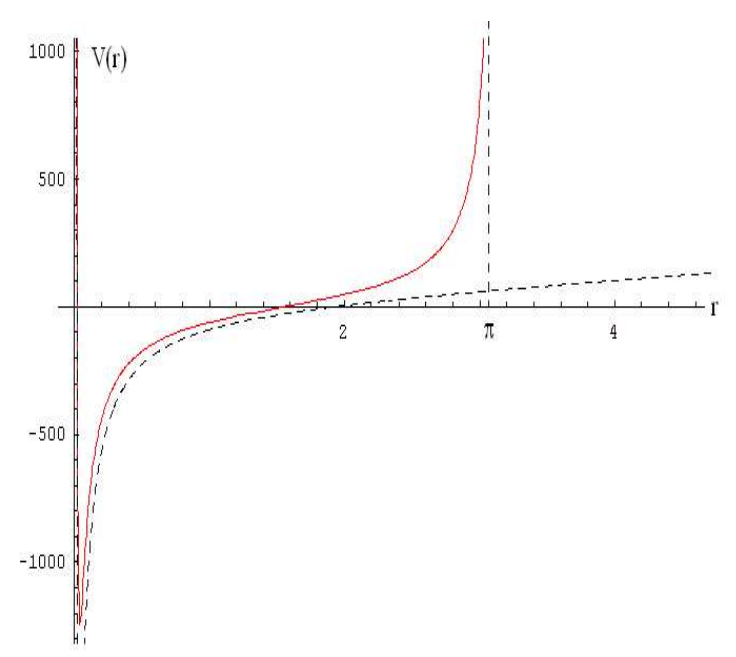

Fig. 1. The trigonometric Rosen-Morse potential (solid line) and its proximity to the Coulomb- plus linear potential (dashed line) for the toy values $a=1, b=50$ of the parameters.

given in terms of dimensionless argument and parameters. The figure shows that $v_{\mathrm{tRM}}$ interpolates between the Coulomb-like potential (associated with the one-gluon exchange) and the infinite well. The latter provides the adequate scenario for the asymptotic freedom insofar as it describes trapped but free particles. The intermediate region of the by and large linear confinement potential can be attributed, as usual, to gluon flux tube links between the quarks. The length scale, $d$, will be considered as a free parameter to be fitted to data. The Taylor expansion of the $\csc ^{2}$ term reads,

$$
a(a+1) \csc ^{2}|\mathbf{z}| \approx \frac{a(a+1)}{\mathbf{z}^{2}}+\frac{a(a+1)}{15} \mathbf{z}^{2} .
$$

The parameter $a$ can be viewed as relative angular momentum in the case when it takes integer non-negative values, a parametrization used in ref. [6]. If so, the $\csc ^{2}$ term acquires meaning of a non-standard centrifugal barrier.

We were able to trace back $v_{\text {tRM }}$ to ref. [7] where it apparently has been found for the first time as a pure mathematical construct while completing the SUSYQM compilation of shape invariant potentials that are exactly solvable by means of the hypergeometric differential equation. The $v_{\text {tRM }}$ potential is therefore one of the new potentials predicted by SUSYQM [5]. We here suggest that the $Q Q$ interactions are ruled by the following trigonometric quark confinement (TQC) potential:

$$
\begin{aligned}
& V_{3 Q}^{\mathrm{TQC}}\left(\mathbf{z}_{1}, \mathbf{z}_{2}, \mathbf{z}_{\mathbf{3}}\right)=-2 b \sum_{i<j} \cot \left|\mathbf{z}_{i}-\mathbf{z}_{j}\right| \\
& +a(a+1) \sum_{i<j} \csc ^{2}\left|\mathbf{z}_{i}-\mathbf{z}_{j}\right|, \quad \mathbf{z}_{i}=\frac{\mathbf{r}_{i}}{d} .
\end{aligned}
$$

The great advantage of the TQC potential over the Coulomb- plus linear potential (compared in fig. (1)) is that while the latter is neither especially symmetric, nor exactly solvable, the former is both, it is exactly solvable, and has in addition the dynamical $O(4)$ symmetry [6] for $a=l=0,1,2, \ldots$, and $S O(2,1)$ for more general $a$ values [8].

\section{The exact single-particle basis of $v_{t R M}$}

In this section we briefly review, for the sake of completeness of the presentation, the exact solutions of the three-dimensional single-particle Schrödinger equation with $v_{\mathrm{tRM}}$, as it appears in the quark-diquark approximation to nucleon structure $[6,9]$. From here onward we shall identify the $a$ parameter with the relative quark-diquark angular momentum. The above equation is solved in the usual way in separating variables using the ansatz

$$
\Psi_{(K l m)}(\mathbf{z})=Y_{(l m)}(\theta, \varphi) \frac{\mathcal{R}_{(K l)}(|\mathbf{z}|)}{|\mathbf{z}|} .
$$

The exact solutions of the radial part have been constructed in ref. [6] on the basis of the one-dimensional solutions found in [9] as

$$
\begin{aligned}
& \mathcal{R}_{(K l)}(|\mathbf{z}|)=N_{(K l)} \sin ^{K+1}|\mathbf{z}| e^{-\frac{b|\mathbf{z}|}{K+1}} R_{K-l}^{\left(\frac{2 b}{K+1},-(K+1)\right)}(\cot |\mathbf{z}|), \\
& K=0,1,2, \ldots, \quad l=0,1, \ldots, K
\end{aligned}
$$

Here, $N_{(K l)}$ is a normalization constant and $\mathbf{z}$ is the relative quark-diquark distance. The $R_{n}^{(\alpha, \beta)}(\cot |\mathbf{z}|)$ functions are the non-classical Romanovski polynomials [10, 11] which are defined by the following Rodrigues formula:

$$
\begin{aligned}
R_{n}^{(\alpha, \beta)}(x)= & e^{\alpha \cot ^{-1} x}\left(1+x^{2}\right)^{-\beta+1} \\
& \times \frac{\mathrm{d}^{n}}{\mathrm{~d} x^{n}} e^{-\alpha \cot ^{-1} x}\left(1+x^{2}\right)^{\beta-1+n},
\end{aligned}
$$

where $x=\cot |\mathbf{z}|$ (see ref. [12] for a recent review). The energy spectrum of the TQC potential is calculated as

$$
\epsilon_{K}=-b^{2} \frac{1}{(K+1)^{2}}+(K+1)^{2}, \quad K=n+l,
$$

where $n=0,1,2, \ldots$ Therefore, particular quark levels bound within different TQC potentials (distinct by the values of $l$ ) happen to carry same energies and align to series of states (multiplets) characterized by the superior quantum number $K$ (see fig. 2). One easily recognizes that the energy in eq. (10) is defined by the Balmer term and its inverse of opposite sign, thus revealing $O(4)$ as dynamical symmetry of the problem. Correspondingly, the $K$-series belong to the irreducible $O(4)$ representations of the type $\left(\frac{K}{2}, \frac{K}{2}\right)$ and $K$ has the meaning of four-dimensional angular momentum. Upon coupling the quark spin to them, one finds the reducible $O(4)$ multiplet $\left(\frac{K}{2}, \frac{K}{2}\right) \otimes\left(\frac{1}{2}, 0\right) \oplus\left(0, \frac{1}{2}\right)$, which consists of the $K$ parity mates $\frac{1}{2}^{ \pm}, \ldots,\left(K-\frac{1}{2}\right)^{ \pm}$, and the one unpaired state of maximal spin $J_{\max }=K+\frac{1}{2}$ of either positive, or, negative parity. 


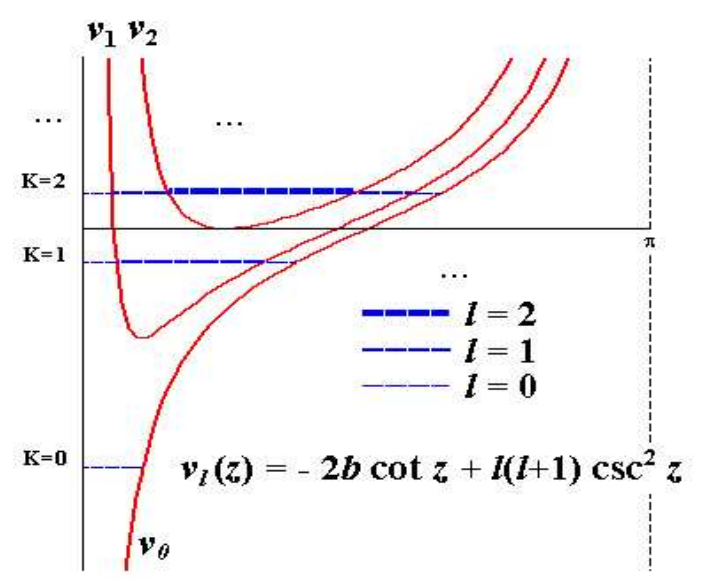

Fig. 2. Schematic presentation of the lowest- $K$ levels in eq. (8). They unite states of same energies from different TQC potentials, here denoted by $v_{l}$, whose angular momenta vary according to the rule $l=0,1,2, \ldots, K$.

\subsection{The nucleon spectrum}

The comparison of the TQC spectrum in fig. 2 to the nucleon excitations reported by [13] reveals an amazing coincidence. All the observed nucleon resonances with masses below $2.5 \mathrm{GeV}$ do indeed fall into $K=1,3,5$ multiplets from which only the $F_{17}$ and $H_{1,11}$ states are still "missing", an observation due to refs. [14]. The $K=2,4$ levels have been attributed to entirely "missing" resonances. The unnatural parity of the $K=3,5$ states would require to account of the internal structure of the diquark. This phenomenon can be interpreted as dominance of a quarkdiquark configuration in nucleon structure (see last reference in [14] for more details) meaning that i) eq. (10) can be directly employed to fit data on the nucleon spectra, ii) matrix elements of transition operators can be evaluated in the basis of eq. (8). The nucleon spectrum is fitted by the following potential parameters [6] :

$$
b=5.85, \quad d=2.31 \mathrm{fm}, \quad \mu=1.06 \mathrm{fm}^{-1},
$$

where $\mu$ stands for the reduced mass of the quark-diquark system. The wave function of the nucleon ground state, $K=0, l=0$, is obtained as

$$
\mathcal{R}_{(00)}(|\mathbf{z}|)=N_{0} e^{-b|\mathbf{z}|} \sin |\mathbf{z}|, \quad N_{0}^{2}=\frac{4 b\left(b^{2}+1\right)}{1-e^{-2 \pi b}} .
$$

In order to illustrate the predictive power of the TQC potential and the efficiency of its exact single-particle basis we shall exploit in the next section the potential parameters in eq. (11) and the wave function in eq. (12) in the calculation of the proton electric form-factor in the quarkdiquark picture of baryon structure.

\section{The proton charge form factor}

The electric form factor is defined in the standard way [15] as the matrix element of the charge component, $J_{0}(\mathbf{r})$,

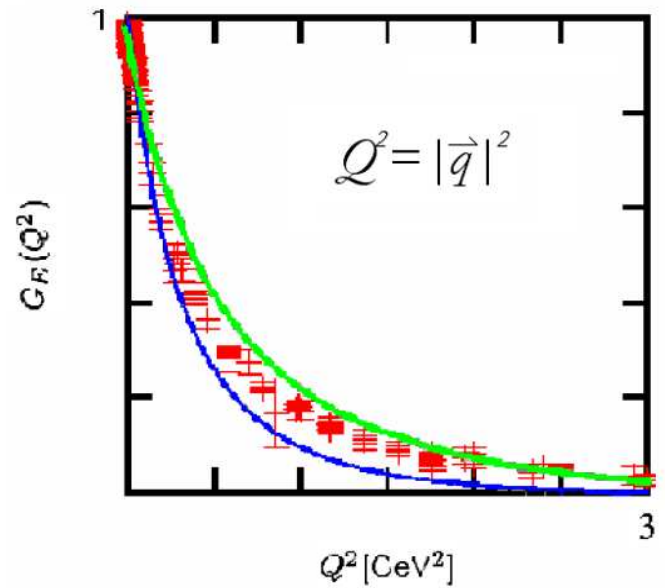

Fig. 3. Comparison of the proton electric form factor as obtained from the analytic expression in eq. (15) (upper curve), and the numerical solution of the Bethe-Salpeter equation (lower curve) reported in ref. [16]. In elastic electron-proton scattering, $Q^{2}=-q^{2}=-q_{0}^{2}+\mathbf{q}^{2}$, and $q_{0}=0$. The experimental data set is same as in [16].

of the proton electric current between the states of the incoming, $\mathbf{p}_{i}$, and outgoing, $\mathbf{p}_{f}$, electrons in the dispersion process,

$$
G_{\mathrm{E}}^{\mathrm{p}}(|\mathbf{q}|)=\left\langle\mathbf{p}_{f}\left|J_{0}(\mathbf{r})\right| \mathbf{p}_{i}\right\rangle, \quad \mathbf{q}=\mathbf{p}_{i}-\mathbf{p}_{f} .
$$

The explicit evaluation of eq. (13), with $J_{0}(\mathbf{r})=$ $e_{\mathrm{p}}\left|\psi_{\mathrm{gst}}(\mathbf{r})\right|^{2}, e_{\mathrm{p}}=1$, and plane waves for the electron states amounts to the calculation of the following integral:

$$
G_{\mathrm{E}}^{\mathrm{p}}(|\mathbf{q}|)=\int_{0}^{\pi d} \mathrm{~d}|\mathbf{r}| \frac{\left(\mathcal{R}_{(00)}(|\mathbf{r}|)\right)^{2} \sin |\mathbf{q}||\mathbf{r}|}{|\mathbf{q}||\mathbf{r}|}
$$

In the $\int_{0}^{\pi d} \longrightarrow \int_{0}^{\infty}$ limit, the integral calculates exactly and is given in terms of $\widetilde{\mathbf{q}}=\mathbf{q} d$ as

$$
\begin{aligned}
& G_{\mathrm{E}}^{\mathrm{p}}(|\widetilde{\mathbf{q}}|)=\frac{b\left(b^{2}+1\right)}{|\widetilde{\mathbf{q}}|} \\
& \times \tan ^{-1} \quad \frac{16|\widetilde{\mathbf{q}}| b}{\widetilde{\mathbf{q}}^{4}+4\left(2 b^{2}-1\right) \widetilde{\mathbf{q}}^{2}+16 b^{2}\left(b^{2}+1\right)} .
\end{aligned}
$$

The exact electric form factor of the proton obtained from eq. (15) is displayed in fig. 3 and follows pretty well the experimental data.

\section{Effective gluon propagator}

The proximity of the TQC potential to the QCD quarkgluon dynamics is suggestive of the idea to exploit the Born approximation and introduce an effective gluon propagator as a Fourier transform of that very potential. In so doing one encounters the integral

$$
\Pi(|\mathbf{q}|)=-\frac{1}{4 \pi} \frac{2 \mu}{\hbar^{2}} \int \mathrm{d}^{3} \mathbf{r} e^{i \mathbf{q} \cdot \mathbf{r}} V(\mathbf{r}),
$$

which, unfortunately diverges. As a remedy, we consider instead the Fourier transforms of potential matrix elements and find integrals that can be taken in closed form. 


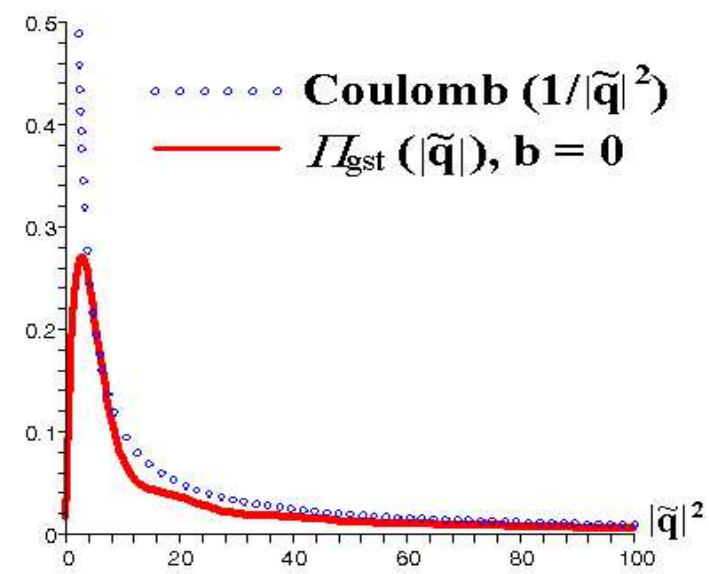

Fig. 4. Comparison of the Coulomb propagator (dotted line) with the TQC-potential propagator for $b=0$ and normalized ground-state wave function (solid line).

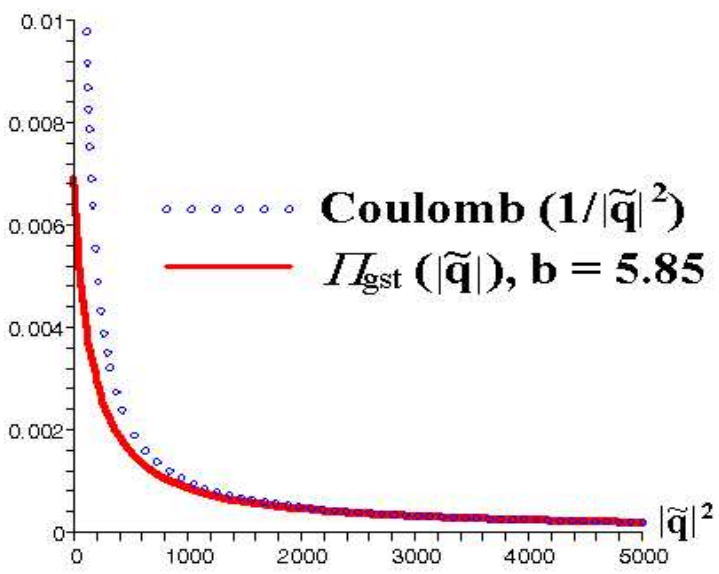

Fig. 5. Comparison of the Coulomb propagator (dotted line) with the TQC-potential propagator for $b=5.85$ and unnormalized ground-state wave function (solid line).

Specifically, the Fourier transform of the ground-state matrix element is calculated as

$$
\begin{aligned}
\Pi^{\text {(gst) }}(|\mathbf{q}|) & =\int_{0}^{\pi d} \mathrm{~d}|\mathbf{r}| \frac{\sin |\mathbf{q}||\mathbf{r}|}{|\mathbf{q}||\mathbf{r}|} v_{\mathrm{tRM}}(|\mathbf{r}|) \psi_{\mathrm{gst}}^{2}(\mathbf{r}) \mathbf{r}^{2} \\
& =N_{(0,0)}^{2} \int_{0}^{\pi} \mathrm{d} y \frac{e^{-2 b y} \sin ^{2} y \sin q^{\prime} y \cot y}{q^{\prime} y}
\end{aligned}
$$

which expresses in terms of $E i(u)=\int_{1}^{\infty} \frac{e^{-u t}}{t} \mathrm{~d} t$, and $C i(u)=\gamma+\ln u+\int_{0}^{u} \mathrm{~d} t \frac{\cos t-1}{t}$ functions, where

$$
y \equiv|\mathbf{z}|=\frac{|\mathbf{r}|}{d}, \quad q^{\prime} \equiv|\widetilde{\mathbf{q}}|=\sqrt{Q^{2}} d
$$

In figs. 4,5 we display $\Pi^{\text {(gst) }}(|\mathbf{q}|)$ for various values of the $b$ parameter. The convenience of our consideration is supported by the great similarity in the asymptotic behavior of the Coulomb propagator and $\Pi^{\text {(gst) }}(|\mathbf{q}|)$.

\section{Concluding remarks}

In this work we pointed out that the trigonometric RosenMorse potential captures quite realistically the traits of the QCD quark-gluon dynamics. This is reflected by the facts that $i)$ the ratio of $(-1 / 3)$ of the strengths of the linear to Coulomb term following from the Taylor expansion of the TQC potential is in line with the value of $(-1 / 2)-(-1 / 4)$ calculated by lattice QCD, ii) the prediction of the proton electric form factor in closed form follows quite satisfactory data, iii) the nucleon spectrum is well reproduced (and the $\Delta$ one as well, skipped out here), iv) the effective gluon propagator obtained as the Fourier transform of the ground-state matrix element of the TQC potential has reasonable shape and asymptotic behavior. The exact single-particle basis of the TQC potential seems quite efficient, indeed, and provides a good starting point for more detailed spectroscopic studies. The TQC potential can be enriched, for example, by the inclusion of $\boldsymbol{\sigma}_{i} \cdot \boldsymbol{\sigma}_{j}$ interactions to account for the spin dynamics, and by screening effects along the line of, say, ref. [3]. The effective gluon propagator can be employed in studies of non-perturbative QCD phenomena in the spirit of ref. [17]. In conclusion, the single-particle basis of the TQC potential considered here seems to provide an efficient tool for quark model calculations of spectroscopic characteristics of baryons.

\section{References}

1. H.J. Rothe, Lattice Gauge Theories (World Scientific, Singapore, 1992).

2. T.T. Takahashi, H. Suganuma, Y. Nemoto, H. Matsufuru, Phys. Rev. D 65, 114509 (2002).

3. P. González, J. Vijande, A. Valcare, H. Garcilazo, Eur. Phys. J. A 29, 235 (2006).

4. E. Santopinto, Phys. Rev. C 72, 022201(R) (2005).

5. R. De, R. Dutt, U. Sukhatme, J. Phys. A: Math. Gen. 25, L843 (1992).

6. C.B. Compean, M. Kirchbach, Bled Workshops in Physics, Vol. 7, No. 1 (DMFA-Zaloznistvo, Ljubljana, 2006) pp. 7-19; E-Print Archive: quant-ph/0610001.

7. G. Levai, J. Phys. A: Math. Gen. 22, 689 (1989).

8. C. Rasinariu, J.V. Mallow, A. Gangopadhyaya, Cent. Eur. J. Phys. 5(2), 111 (2007); R. Koc, M. Koca, J. Phys. A: Math. Gen. 36, 8105 (2003).

9. C.B. Compean, M. Kirchbach, J. Phys. A: Math. Gen. 39, $547(2006)$

10. E.J. Routh, Proc. London Math. Soc. 16, 245 (1884).

11. V. Romanovski, C. R. Acad. Sci. Paris, 188, 1023 (1929).

12. A. Raposo, H.J. Weber, D.E. Alvarez-Castillo, M. Kirchbach, Cent. Eur. J. Phys. 5(3), 253 (2007).

13. S. Eidelman et al., Phys. Lett. B 592, 1 (2004).

14. M. Kirchbach, Mod. Phys. Lett. A 12, 2373 (1997); Int. J. Mod. Phys. A 15, 1435 (2000); M. Kirchbach, M. Moshinsky, Yu. F. Smirnov, Phys. Rev. D 64, 114005 (2001).

15. J. Arrington, C.D. Roberts, J.M. Zanotti, J. Phys. G: Nucl. Part. 34, S23 (2007).

16. Chr. Haupt, www.itkp.uni-bonn.de/ haupt/talks/ Internal_2005.pdf, and references therein.

17. F.T. Hawes, C.D. Roberts, A.G. Williams, Phys. Rev. D 49, 4683 (1994). 